\title{
Accueillir la diversité des apprenants. Agencer la pluralité des modes d'enseignement
}

\section{Marisa Cavalli}

\section{(2) OpenEdition \\ Journals}

Édition électronique

URL : http://journals.openedition.org/trema/3264

DOI : $10.4000 /$ trema.3264

ISSN : 2107-0997

Éditeur

Faculté d'Éducation de l'université de Montpellier

Édition imprimée

Date de publication : 1 novembre 2014

Pagination : 20 - 31

ISSN : 1167-315X

Référence électronique

Marisa Cavalli, «Accueillir la diversité des apprenants. Agencer la pluralité des modes

d'enseignement », Tréma [En ligne], 42 | 2014, mis en ligne le 16 juin 2015, consulté le 20 avril 2019.

URL : http://journals.openedition.org/trema/3264; DOI : 10.4000/trema.3264

Ce document a été généré automatiquement le 20 avril 2019

Trema 


\title{
Accueillir la diversité des apprenants. Agencer la pluralité des modes d'enseignement
}

\author{
Marisa Cavalli
}

1 Les réflexions qui suivent porteront, dans un premier temps, sur les raisons qui justifient et argumentent l'enseignement plurilingue à l'école primaire, étant bien entendu que ce type d'enseignement recouvre les situations éducatives les plus différentes pouvant avoir les finalités les plus diversifiées (enseignement de la langue de scolarisation et d'une langue étrangère, sauvegarde de langues régionales ou minoritaires, section européennes, sections bilingues, enseignement des langues d'origine aux côtés de la langue de scolarisation, prise en compte des répertoires de départ des apprenants, sensibilisation à la pluralité et à la diversité linguistique et culturelle ...). Nous aborderons, dans un deuxième temps, l'analyse des implications de la didactique du plurilinguisme à l'école primaire en fonction de la diversité des contextes institutionnels et culturels et fournirons, pour conclure, quelques suggestions pour la mise en œuvre concrète d'un enseignement plurilingue contextualisé exploitant la multiplicité des approches existantes.

\section{Plurilinguisme à l'école : une nouvelle idéologie?}

Certains ouvrages et articles, plus ou moins récents (entre autres, Maurer, 2001 ; Canut et Duchêne, 2011; Véronique, 2005) et certains de leurs commentateurs questionnent, de façon plus ou moins polémique, les notions de plurilinguisme et d'éducation plurilingue et interculturelle sur lesquelles s'appuient les politiques linguistiques éducatives, notamment et récemment celles du Conseil de l'Europe.

Il est reproché à ces dernières de pécher par «angélisme ", si ce n'est carrément, dans certains positionnements, par complicité avec un vaste complot néolibéral, dont une des visées serait la destruction $d u$ «bon » enseignement d'antan (dont on aimerait bien que 
soient explicitées, par ailleurs, les qualités $\left.{ }^{1}\right)$. Le reproche le plus courant étant que les preuves scientifiques de l'efficacité de l'enseignement plurilingue manquent à l'appel.

Étant posé que toute critique est salutaire quand elle est fondée scientifiquement et que la déstabilisation qu'elle provoque est utile à la validation ou à l'invalidation de toute théorie (dans l'optique poppérienne), le fait que ce genre de critiques proviennent essentiellement du domaine académique francophone ne peut que laisser rêveur. À se demander si ce ne sont là de profondes traces laissées par le jacobinisme linguistique lié à la langue française...

Car les gens du terrain ayant depuis longtemps travaillé dans des situations scolaires plurilingues peuvent se demander s'ils vivent sur la même planète que ces chercheurs. Car eux, qui ont les expériences, directes et quotidiennes, des effets de l'enseignement plurilingue sur les enfants, qui connaissent aussi bien les difficultés que les atouts qu'il offre, eux qui ne vivent pas que de certitudes béates et militantes, ils ont cherché des confirmations scientifiques à leurs pratiques et ils en ont trouvé foison. Il est vrai, plutôt du côté de la recherche en langue anglaise.

6 Et dans les domaines les plus variés (didactique des langues, psychologie, psycholinguistique, neurologie, neurosciences, etc.). Soulignons que souvent cette recherche de confirmations se fait moins dans une optique offensive (mettre en avant les arguments en faveur du plurilinguisme) que dans une optique défensive (trouver des réponses aux doutes et aux craintes - des parents, des médias, parfois des autorités doutes et craintes propres à chacun) et que le souci majeur est l'assurance que les meilleurs choix éducatifs et didactiques ont été faits en faveur de l'élève. Nous reviendrons sur ce point plus loin.

7 Le plurilinguisme à l'école serait-il donc une nouvelle idéologie ? Pourquoi pas, si l'on admet que certaines critiques à son encontre relèvent elles aussi d'une idéologie toute contraire. Pourquoi pas, si par idéologie on entend un « ensemble plus ou moins cohérent des idées, des croyances et des doctrines philosophiques, religieuses, politiques, économiques, sociales, propre à une époque, une société, une classe et qui oriente l'action $»^{2}$ et non pas son acception péjorative. Pourquoi pas, si dans «idéologie » on relève la racine de mots tels que « idée », « idéalisme », « idéal » et surtout « idéer ».

\section{Que le plurilinguisme n'est pas qu'une idée}

8 Or, il s'avère - et les enseignants le constatent dans leurs classes - que le plurilinguisme des locuteurs et le multilinguisme des espaces ne sont pas une situation extraordinaire, si tant est qu'ils l'aient jamais été. Les phénomènes si souvent évoqués dans des sortes de litanies - changements démographiques actuels avec augmentation des flux migratoires, mondialisation de l'économie, nouvelles technologies, accroissement des échanges virtuels et réels dans un monde de plus en plus interconnecté - rendent beaucoup plus visible et spectaculaire une situation qui a existé de tout temps, de façon plus ou moins ténue ou intense, selon les lieux et les époques : la diversité linguistique et culturelle.

Un des problèmes majeurs réside dans le fait que le monde académique et, à sa suite, les systèmes éducatifs ont, dans le temps, contribué à formater un regard monolingue sur des réalités sociales où la pluralité et la diversité ont toujours été présentes, ne serait-ce, que, dans le cas limite, dans la variation interne de toute langue pour nationale et standard qu'on l'ait voulue et conçue. 
10 Sans compter la volonté politique de monolinguisme des États-nations qui entendait effacer la complexité de la réalité.

11 Serait-on donc revenus, dans les écrits évoqués plus haut, à la perspective « language as problem» (Baker and Pys Jones, 1998: 276 - 286) ou au plurilinguisme «en procès » (Tabouret-Keller, 2011)?

12 Au-delà des débats d'idées, nous nous plaçons ici dans la perspective concrète de l'élève et de l'enseignant(e) dans la classe dans des situations bien réelles de plurilinguisme.

Que faire donc face à cette évidence?

\section{L'éducation plurilingue et interculturelle : aboutissement d'un changement de paradigme}

13 La notion d'éducation plurilingue et interculturelle ${ }^{3}$ est une réponse didactique, parmi d'autres possibles, aux besoins de l'école et de ses acteurs principaux, les élèves. Elle est, en même temps, prise en compte et mise en valeur du multilinguisme sociétal et du plurilinguisme individuel ainsi que leur traduction éducative et formative.

14 Cette notion est le fruit d'un changement de paradigme qui s'est opéré, au fil du temps, dans la didactique des langues, par une série de déplacements qui l'ont profondément changée et qui ont contribué à l'évolution de la conception monolingue traditionnelle des apprentissages des langues vers une conception plurilingue.

La didactique des langues s'est dans le temps enrichie de nouvelles approches qui s'appuient sur un nombre plus ou moins élevé de langues (approches plurielles) et qui prennent appui sur une série de basculements représentationnels, que nous listerons en 10 points :

16 1. d'une conception cloisonnée / cloîtrée des langues de l'école (sorte de huis-clos scolaire) à une conception écologique plus large prenant en compte les langues des répertoires des apprenants, les langues de l'environnement proche et les langues enseignées à l'école ;

17 2. d'une didactique "mono » centrée (touchant une langue à la fois) et séparant les langues en domaines nettement différenciés et sans rapports les uns avec les autres et caractérisés par des didactiques différentes (langues étrangères vs langues régionales / minoritaires vs langues de scolarisation vs langues classiques) à une conception décloisonnée, holistique des langues qui, sans nier les différences de leurs statuts cognitifs et leurs implications didactiques (Vigner, 2009 ; Verdelhan, 2007) :

- assume la complexité de la question des langues à l'école en considérantensemble :

- les langues des répertoires des apprenants (locuteurs de langues régionales / minoritaires, de la migration, de variétés - non standard / non légitimes à l'école - de la langue de scolarisation) même quand elles ne font pas l'objet d'un enseignement ;

- la/les langue/s de scolarisation dans leur double dimension : langue enseignée comme matière et langue utilisée dans les autres matières,

- les langues enseignées par l'école : étrangères et classiques ;

- les langues régionales ou minoritaires ou de la migration quand elles font l'objet d'un enseignement scolaire ;

- et s'appuie sur une didactique intégrée des langues pour rationaliser, coordonner et seconder les apprentissages langagiers et culturels : par la mise en place de ponts, de 
passerelles entre les langues, par l'entraînement à des stratégies de transfert, par le développement de capacités d'ordre « méta » par rapport aux langues et à leur apprentissage, par le repérage systématique de similitudes et différences entre les langues, ...;

3. d'une vision fonctionnelle et utilitaire de l'enseignement - orientée essentiellement vers la maitrise langagière et communicative - à une conception plus largement formatrice des langues, ces dernières étant conçues

- aussi bien comme des valeurs que comme véhiculant (dans le sens de «permettant de construire ») des valeurs, telles que la citoyenneté démocratique, l'inclusion sociale, le respect de la diversité et de la pluralité des langues et des cultures, la bienveillance ;

- comme étant propices à la formation de l'individu et du futur citoyen (cf. educazione linguistica, Costanzo, 2003), éducation langagière globale, éducation plurilingue et interculturelle (Conseil de l'Europe, 2009), éducation à la pluralité des langues et des cultures) ;

- comme vecteurs / moyens de construction des connaissances autres que linguistiques, par la prise en compte du rôle des dimensions langagières dans l'enseignement - apprentissage des autres matières ; 4. d'une vision centrée sur la seule acquisition à l'école d'une compétence approfondie dans toutes les activités langagières (réception, expression et médiation orales et écrites) à également une sensibilisation à et un « brassage » de plusieurs langues, se situant à côté de l'enseignement traditionnel, pour :

- une éducation langagière globale propédeutique aux enseignements - apprentissages plurilingues et interculturels, avec valorisation des répertoires langagiers et culturels des apprenants ;

- l'acquisition d'une seule activité langagière (voire plus d'une) dans une ou plusieurs langues (compétences partielles mais approfondies, par exemple de lecture);

5. d'une vision irréaliste de la langue ayant le locuteur natif comme modèle à une vision plus réaliste $\mathrm{du}$ répertoire plurilingue comme d'une construction progressive d'approximation en approximation - dans laquelle l'erreur joue un rôle révélateur de processus mentaux intra- et interlinguistiques complexes, avec la conscience de l'instabilité relative et dynamique des compétences acquises dans les divers systèmes linguistiques suivant la fréquence de leur emploi, les domaines d'utilisation, ...;

6. d'une vision du fonctionnement discursif dans la classe en mode uniquement monolingue à une adoption (et valorisation) de fonctionnements en mode bi-/ plurilingue (alternance des langues et alternance entre les divers modes (mono - bi - pluri) au moyen du contrat didactique $\left.{ }^{4}\right)$;

7. d'une vision de l'école comme sanctuaire de la norme à une vision de l'école comme lieu où la variabilité interne de toute langue (y compris, surtout et avant tout, de la langue de scolarisation) y est objet d'étude, d'expérimentions et de gestion;

8. d'une vision cloisonnée et concurrente des diverses approches didactiques à une intégration de ces approches et à leur utilisation flexible - en complémentarité - pour répondre aux besoins différenciés de l'enseignement - apprentissage selon les contextes ;

9. d'une conception de la didactique des cultures visant des connaissances dans les cultures de l'autre langue à des conceptions se donnant comme finalité l'éducation à l'altérité (Abdallah Preitchelle, 1999 \& 2003 ; Byram, 2008) jusqu'à la reconnaissance de l'altérité en soi (Blanchet \& Coste, 2010); 

d'établissement et celle de la construction curriculaire, comme moyens, la deuxième s'imbriquant dans la première, pour assurer la finalité essentielle de l'école : garantir à tous les élèves leur droit à une éducation de qualité dont la maîtrise des langues représente une condition incontournable (Coste et alii, 2009).

\section{1 Une politique linguistique d'établissement}

31 Pour faire face aux changements sociétaux énumérés plus haut et pour, notamment, prendre activement en compte la configuration toute particulière qu'ils assument dans chaque contexte spécifique, il importe de penser en termes de politique linguistique d'établissement. Non pas en tant que seule prise en compte de l'enseignement des langues, mais en tant que les langues représentent une dimension constitutive et transversale à tous les domaines de l'éducation et de l'école.

Nous prônons l'élaboration d'une politique linguistique qui concernerait tous les acteurs de l'établissement :

- en tout premier lieu les destinataires du service scolaire : les apprenants et leurs familles qu'il s'agit d'informer, d'impliquer dans des collaborations et avec lesquelles il importe d'établir un dialogue authentique et constructif ${ }^{5}$, y compris à propos des langues, de leur emploi et enseignement et à propos de la politique linguistique d'établissement ;

Tréma, 42 | 2015 
- les enseignant(e)s - aussi bien de langues que des autres matières scolaires - puisque tou(te)s sont responsables, chacun(e) dans sa propre discipline, de l'élargissement et de l'enrichissement du répertoire langagier des apprenants ;

- les collaborateurs / médiateurs ou autres intervenants à côté des enseignants ; tous les personnels autres de l'école ;

- et, bien entendu, le chef d'établissement dont on ne soulignera jamais assez le rôle tout à fait central qu'il peut jouer, en tant que catalyseur de l'innovation, pour valoriser les ressources humaines que constituent les personnels d'une école, pour les aider dans leur travail, pour les motiver, les guider, les responsabiliser et les autonomiser.

Si la politique linguistique d'établissement doit tenir compte des contraintes et des opportunités venant du niveau national, elle doit pouvoir s'adapter aux caractéristiques et répondre aux besoins spécifiques de l'environnement socioculturel et sociolinguistique sur lequel l'établissement agit directement.

Le protocole pour élaborer une politique linguistique d'établissement pourrait ainsi suivre les étapes suivantes ${ }^{6}$ :

- analyser les besoins linguistiques et langagiers du contexte environnant (du tissu social dans son ensemble, des travailleurs, des communautés présentes, ...) et individuellement des élèves ;

- s'appuyer pour cela sur des données : utiliser celles qui sont disponibles et se donner les moyens pour obtenir celles qui sont indispensables ; les renouveler et les accroître progressivement d'année en année pour permettre d'adapter, dans les limites du possible, la politique linguistique d'établissement aux changements intervenant au fur et à mesure dans la société :

- savoir bien analyser ces données et les prendre en compte pour établir les actions adaptées de façon à répondre aux divers besoins relevés ;

- repérer toutes les ressources disponibles, qu'elles soient normatives, internes à l'établissement ou bien externes ;

- se donner des objectifs réalistes mais progressifs pour la politique linguistique d'établissement, tout en faisant preuve de conviction et de créativité ;

- envisager les mesures à mettre en œuvre qui impliquent directement tout le personnel de l'école (espaces de communication et d'échange, espaces de collaboration, projets, ateliers, cours de langues, échanges avec le territoire, avec d'autres écoles, à l'étranger ...) ;

- prévoir les dispositifs d'évaluation des objectifs de politique linguistique d'établissement fixés de façon à saisir concrètement les avancements ;

- mettre en musique tout ce qui précède dans un document officiel qui argumente la politique linguistique de l'établissement et en détaille les mesures.

La politique linguistique d'établissement ne devrait pas constituer un document de plus dont les effets se termineraient avec sa rédaction : elle devrait être la source d'inspiration continuelle pour tous ses agents, le fil rouge de l'action commune et des actions individuelles. Elle contribuerait à caractériser l'identité propre de chaque établissement. Car assumer transversalement les langues signifie toucher à tous les aspects de l'éducation : la pluralité, la diversité et l'altérité sous toutes ses formes (origine sociale, ethnique, genre, religion ...), leur accueil et respect, la gestion des conflits, mais aussi les valeurs à défendre, les compétences à construire et les modalités à suivre pour ce faire, les expériences à faire vivre, les évaluations des progrès à fournir. 


\section{2 La construction curriculaire}

Les orientations de la politique linguistique d'établissement demeurent encore assez générales : pour être actives, elles doivent se concrétiser dans le curriculum, entendu ici comme le parcours d'apprentissage que l'école offre à l'apprenant.

L'élaboration d'un curriculum d'éducation plurilingue et interculturelle s'appuie sur les programmes nationaux et s'insère dans le cadre des orientations de la politique linguistique d'établissement.

Le curriculum détaille les objectifs en termes de compétences à atteindre :

- verticalement, c'est-à-dire par cycles / paliers dans les diverses langues et disciplines;

- horizontalement, c'est-à-dire par année scolaire, dans :

- la/les langue/s de scolarisation;

- la langue régionale ou minoritaire ou de la migration dans les cas où elles sont enseignées ;

- la langue étrangère.

Pour la langue de scolarisation (mais aussi pour toute autre langue utilisée dans l'acquisition de connaissances dans les matières), le curriculum spécifie les compétences à $\mathrm{y}$ atteindre aussi bien en tant que langue comme matière (perfectionnement de l'expression orale, lecture, écriture, littérature pour les jeunes...) que comme langue utilisée pour les apprentissages des autres matières (mathématiques, histoire, science...).

Comme l'apprentissage se réalise efficacement à travers la possibilité offerte à l'élève de vivre des expériences significatives et diverses, le curriculum gagnerait à être pensé aussi en termes d'expériences d'apprentissage pertinentes par rapport à l'éducation plurilingue et interculturelle 7 . Parmi ces expériences, par exemple, doivent trouver place la valorisation et la prise en compte des langues des répertoires des apprenants, mais aussi les échanges virtuels ou réels avec des élèves d'autres classes, étrangères ou pas.

$41 \mathrm{Au}$ cours de la construction curriculaire, on repèrera, dans la panoplie des approches disponibles, celles qui peuvent le mieux répondre aux besoins spécifiques d'un certain contexte et, dans celui-ci, de certains (groupes d') apprenants. Si, pour ces deniers, on pourra mettre en place des mesures d'individualisation et de différenciation, il faudrait avoir constamment à l'esprit que prendre en considération les difficultés de certains élèves induit des manières d'enseigner qui profitent à tous les élèves. Le tout premier enseignement étant justement celui de l'accueil et de la valorisation par l'enseignant de chacun, y compris pour ce qui est des ressources langagières et culturelles dont il dispose.

2 Un outil qui pourrait faciliter la construction du curriculum plurilingue est le scénario curriculaire (cf. Beacco et alii, 2010 ; Coste, (éd.) 2013).

\section{Multiplicité des approches - Précautions à prendre}

43 Il s'agit, au moment des choix didactiques, d'avoir conscience que chaque approche est une ressource qui ne convient pas dans n'importe quelle situation et que chacune répond à des exigences et des finalités différentes.

Une didactique bilingue, par exemple, si elle se réalise dans des dispositifs entièrement bilingues, est un choix éducatif fort aux implications complexes et profondes pour les processus de constructions des connaissances et des identités, dont il faut connaître à 
l'avance les coûts en termes financiers, d'organisation, de formation des enseignants, de recherche. C'est un choix ambitieux pour lequel un énorme investissement est indispensable, tout d'abord en termes de conviction, y compris, parfois, politique. Ce n'est donc pas un choix à faire à la légère et sans un engagement de tous les instants et dans la durée. C'est l'erreur que font souvent certains politiques.

Toutefois des formes d'enseignement bilingue sont utilement réalisables dans n'importe quel contexte et rentrent parmi les expériences que tout élève devrait pouvoir vivre à l'école.

La didactique intégrée des langues est une méthodologie de mise en relation des langues connues, enseignées et utilisés à l'école qui devrait permettre, en tout contexte et en toute circonstance, de garantir un enseignement solide, économique, coordonné et, pour cela, renforcé de toutes ces langues. Mais, outre qu'elle représente avant tout un état d'esprit qui devrait informer tout enseignement linguistique dans une optique holistique, cette méthodologie, à partir de principes généraux, valables pour tout contexte, doit se construire - de façon spécifique à chacun - selon les langues en présence, leurs caractéristiques et leurs typologies. Faire de l'intégration linguistique entre français et italien ou entre espagnol et allemand ou entre grec et russe n'a assurément pas les mêmes implications didactiques, mis à part les principes généraux.

Outre le fait qu'il est abordé dans de nombreux ouvrages théoriques de références (cf. Candelier et alii, 2012 pour une bibliographie mise à jour), l'éveil aux langues a, lui, l'énorme avantage de disposer aussi d'une quantité enviable de matériels didactiques, ce qui est un excellent moyen de formation indirecte des enseignants. Par ces matériels réunis sur des sites et dans des publications, facilement adaptables aux caractéristiques des différents contextes, l'éveil aux langues peut remplir diverses fonctions ${ }^{8}$ à l'intérieur d'un curriculum plurilingue, la première par importance étant l'accueil en classe des langues des répertoires des élèves par des activités et des expériences de l'altérité linguistique et culturelle.

L'intercompréhension entre langues (Escudé \& Janin, 2010) - pour lesquelles nous ne ferons pas la distinction entre langues parentes / voisines et langues distantes - est une approche qui - au-delà des mises en œuvre didactiques, pour le moment encore rares pour l'école primaire, à l'exception du manuel Euro-mania (Escudé (dir), 2008) - nous semble devoir être interrogée pour ses apports à tout processus d'appropriation et pour la contribution qu'elle peut fournir à une mise en confiance de l'apprenant face à l'inconnue que constitue toute nouvelle langue à apprendre. L'intercompréhension (bien... comprise) pourrait ainsi contribuer à faire de tout élève un " apprenant curieux " (Py, 1992) faisant recours aux stratégies offensives de prise de risque (Bange, 1993). C'est de la sorte, tout au moins, que cette approche nous paraît particulièrement adaptable à l'enseignement en primaire.

Quant aux approches interculturelles, elles nous paraissent valables dans la mesure où elles permettent de ne pas s'arrêter à l'informationnel et à l'anecdotique et où elles interrogent à fond le rapport individuel à l'altérité, y compris et au-delà de tout angélisme, dans les tensions qu'il peut comporter. Des approches du style Autobiographie des approches interculturelles (Conseil de l'Europe, 2009) accompagnent et structurent un processus individuel de réflexion et de conscientisation de ses propres réactions face aux problématiques complexes de l'altérité et de son respect. 
50 À côté de ces approches, d'autres sont indiquées ailleurs (cf. Beacco et alii, 2010) qui peuvent répondre à des besoins spécifiques de certains contextes en complémentarité d'approches plus « canoniques ».

\section{En guise de non conclusion...}

Dans la construction curriculaire, il ne s'agit nullement de courir tous les lièvres à la fois ou d'innover pour innover ou, pire encore, pour être « à la page ». L'éducation plurilingue et interculturelle est un enjeu fort pour les élèves, pour les adultes et citoyens de demain qu'ils sont en train de devenir. La conscience de leurs besoins concrets en vue d'apprentissages utiles pour leur formation personnelle, pour la suite de leurs études, pour leur avenir personnel et professionnel constitue le meilleur guide à suivre pour fonder des choix pertinents, à situer dans une juste progressivité, suivant la logique des petits pas. Il s'agit pour cela de faire pleinement confiance aux capacités des enseignant (e)s à réaliser le savant dosage et mixage des différentes approches à côté des enseignements visant l'appropriation de chaque langue. Si la formation initiale se doit de leur faire connaître et expérimenter le potentiel de chacune de ces approches, c'est dans l'interaction avec un contexte donné, ses acteurs et leurs caractéristiques, qu'ils/elles pourront sciemment et collectivement faire les choix qui conviennent.

Il nous plait de conclure sur l'importance fondamentale de former les enseignants à la curiosité intellectuelle et de les motiver à leur propre apprentissage tout au long de la vie. Pour cela, il faudrait leur faire prendre conscience que la didactique des langues a été, est et sera toujours, comme toute science qui se respecte, soumise à des évolutions, à des réorientations, à des changements de paradigme, que cela est tout à fait sain et normal pour une science humaine et que toutes ces transformations, quand elles sont justifiées et fondées scientifiquement, font elles aussi partie de l'évolution de l'apprentissage humain.

\section{BIBLIOGRAPHIE}

Abdallah-Pretceille, M. (1999). L'éducation interculturelle. Paris : P.U.F.

Abdallah-Pretceille, M. (2003). Former et éduquer en contexte hétérogène - Pour un humanisme du divers. Paris : Anthropos-Economica.

Baker, C. et Prys Jones, S. (1998). Encyclopedia of Bilingualism and Bilingual Education. Clevedon: Multilingual Matters.

Bange, P. (1992). « À propos de la communication et de l'apprentissage de la L2, notamment dans ses formes institutionnelles ", Acquisition et interaction en langue étrangère (AILE) 1, 53-85.

Beacco, J.-C. et Byram, M. (2007). Guide pour l'élaboration des politiques linguistiques éducatives en Europe - De la diversité linguistique à l'éducation plurilingue, Version intégrale. Strasbourg : Division 
des Politiques linguistiques, Conseil de l'Europe. http://www.coe.int/lang [consulté le 15/03/2013]

Beacco, J.-C., Cavalli, M., Coste, D., Egli Cuenat, M., Goullier, F. et Panthier, J. (2010). Guide pour le développement et la mise en œuvre de curriculums pour une éducation plurilingue et interculturelle. Strasbourg : Division des Politiques linguistiques, Conseil de l'Europe. http://www.coe.int/lang [consulté le 15/03/2013]

Billiez, J. (éd.) (1998). De la didactique des langues à la didactique du plurilinguisme. Hommage à Louise Dabène. Grenoble : CDL-LIDILEM.

Blanchet, P. et Coste, D. (2010). «Sur quelques particularités de la notion d'interculturalité Analyses et propositions dans le cadre d'une didactique de la pluralité linguistique et culturelle », In Blanchet, P. et Coste, D. (dir.) Regards critiques sur la notion d'« interculturalité » - Pour une didactique de la pluralité linguistique et culturelle (pp. 7-27). Paris : L'Harmattan.

Byram, M. (2008). From Foreign Language Education to Education for Intercultural Citizenship Essays and Reflections. Clevedon, Buffalo, Toronto: Multilingual Matters, 2008.

Candelier, M. (coord.), Camilleri-Grima, A., Castellotti, V., De Pietro, J.-F.), Lörincz, I., Meissner, F.-J., Schröder-Sura, A., Noguerol, A. avec le concours de Molinié, M. (2012). CARAP - Cadre de Référence pour les Approches Plurielles des langues et de cultures. Graz : CELV.

Canut, C. et Duchêne, A. (2011). Introduction. Instrumentalisations politiques et économiques des langues : le plurilinguisme en question. Langage et société, 2/201

$\left(n^{\circ} 136\right), 5-12$.

Castellotti, V., Coste, D. et Duverger, J. (coord.) (2008). Propositions pour une éducation au plurilinguisme en contexte scolaire. Paris : ADEB.

Cavalli, M. (éd.), Coste, D., Crisan, A. et Van De Ven, P.-H. (2009). L'éducation plurilingue et interculturelle comme projet. Strasbourg : Conseil de l'Europe. http://www.coe.int/lang [consulté le 15/03/2013]

Commission européenne (2012). First European Survey on Languages Competences - Final report, Education and Training. http://ec.europa.eu/languages/eslc/docs/en/final-report-escl_en.pdf [consulté le 15/03/2013]

Conseil de l'Europe (2009). Plateforme de ressources et de références pour une Éducation plurilingue et interculturelle. Strasbourg : Unité des Politiques linguistiques. http://www.coe.int/lang [consulté le 15/03/2013].

Conseil de l'Europe (2009). Autobiographie des rencontres interculturelles. Strasbourg : Unité des Politiques Linguistiques. http://www.coe.int/lang [consulté le 15/03/2013].

Corson, D. (2008). Language Policy in Schools - A Resource for Teachers and Administrators, 1st edition 1999. New-York-London: Routledge.

Costanzo, E. (2003). L'éducation linguistique (educazione linguistica) en Italie : une expérience pour l'Europe ? Étude de référence, Division des politiques linguistiques - Direction de l'éducation scolaire, extra-scolaire et de l'enseignement supérieur - DG IV - Strasbourg : Conseil de l'Europe. http://www.coe.int/lang [consulté le 15/03/2013]

Coste, D. (éd.) (2013) : Les langues au cœur de l'éducation - Principes, pratiques, propositions. Fernelmont : E.M.E. éditions. 
Coste, D. (éd.), Cavalli, M., Crisan, A. et Van De Ven, P.-H. (2009). L'éducation plurilingue et interculturelle comme droit. Strasbourg : Conseil de l'Europe. http://www.coe.int/lang [consulté le 15/03/2013].

Escudé, P. et Janin, P. (2010). Le point sur l'intercompréhension, clé du plurilinguisme. Paris : Clé International.

Escudé, P. (dir) (2008). J'apprends par les langues - Manuel européen EURO-MANIA. Toulouse : Scéren - CRDP Midi -Pyrénées. http://euro-mania.eu [consulté le 15/03/2013].

Grosjean, F. (1982). Life with Two Languages - An Introduction to Bilingualism. Cambridge, Massachusetts and London, England: Harvard University Press.

Grosjean, F. (2008). Studying Bilinguals. Oxford: Oxford University Press.

Grosjean, F. (2010). Bilingual: Life and Reality. Cambridge, Mass: Harvard University Press.

Heller, M. et Martin-Jones, M. (eds) (2001). Voices of Authority - Education and Linguistic Difference. Westport, Connecticut: Ablex Publishing.

Lüdi, G. et Py, B. (2003). Être bilingue. Ire édition 1986. Berne : Peter Lang.

Maurer, B. (2011). Enseignement des langues et construction européenne. Paris : Edition des archives contemporaines.

Moore, D. (1996). «Bouées transcodiques en situation immersive ou comment interagir avec deux langues quand on apprend une langue étrangère à l'école ». Acquisition et interaction en langue étrangère (AILE), 7, 95-121.

Moore, D. et Simon, D.-L. (2002). « Déritualisation et identité d'apprenants ». Acquisition et interaction en langue étrangère (AILE), 16, 121-144.

Popper, K. R. (1994). Verso una teoria evoluzionistica della conoscenza. Roma: Armando editore.

Py, B. (1993). «L'apprenant et son territoire : système, norme et tâche ». Acquisition et interaction en langue étrangère (AILE), 2, 9-24.

Tabouret-Keller, A. (2011). Le bilinguisme en procès (1840-1940). Limoges : Éditions Lambert Lucas.

Verdelhan-Bourgade, M. (dir.) (2007). Le français langue seconde : un concept et des pratiques en évolution. Bruxelles : De Boeck.

Véronique, D. (2005). «Questions à une didactique de la pluralité des langues ». In M.-A. Mochet et al. (dir.). Plurilinguisme et apprentissages, Mélanges Daniel Coste. Lyon : ENS Éditions.

Vigner, G. (2009). Le français comme langue seconde - Comment apprendre le français aux élèves nouvellement arrivés. Paris : Hachette Education.

\section{NOTES}

1. Surtout au vu des résultats de certaines enquêtes internationales (cf. pour les langues, Commission européenne, Les Européens et leurs langues, enquête Eurobaromètre, 2012).

2. Trésor de la langue français informatisé (www.atilf.fr).

3. Pour une tentative de définition de cette notion, cf. Cavalli et alii, 2009.

4. Ici les travaux suisses (Grosjean, 1982, 2008, 2010 ; Luedi \& Py, 1986/2002) ont ouvert la voie à la réflexion successive sur ce qui se passe en classe. On a beau viser un monolinguisme idéal dans toute classe de langue, la réalité des fonctionnements discursifs montrent que l'alternance des 
langues existe toujours, ne serait-ce que dans les têtes des apprenants, et que mieux vaut la gérer didactiquement que d'en nier académiquement la légitimité. Force est de constater que, malgré le nombre d'études existantes (pour n'en citer que deux : Moore, 1996 ; Moore \& Simon, 2002) y compris sur la gestion et les fonctions des alternances codiques en classe en vue de la construction des connaissances, le mode bi/plurilingue subit le même ostracisme et le même processus de diabolisation que le bi-/plurilinguisme. Pour des exemples, plus ou moins problématiques, d'alternances des langues dans des classes plurilingues, de leurs fonctions et usages, cf. Heller et Martin-Jones (eds), 2001.

5. Deux projets du programme 2012- 2015 du Centre Européen des Langues vivantes se donnent pour but l'implication des parents dans les projets scolaires : l'un Co-construire l'éducation des migrants, coordonné par Andréa Young et l'autre, Implication des parents dans l'éducation plurilingue et interculturelle, coordonné par Stéphanie Clerc, www.ecml.at

6. Pour des orientations similaires, mais plus détaillées, cf. Beacco et Byram, 2007; Beacco et alii, 2010 ; Corson, 2008.

7. On trouvera des listes d'expériences dans les publications suivantes : Castellotti et alii, 2008 ; Beacco et alii, 2010 : 48-53 ; Coste (éd.), (à paraître).

8. Cf. à ce propos, Candelier et alii (2012).

\section{RÉSUMÉS}

Le texte énonce les raisons justifiant l'enseignement plurilingue à l'école primaire, propose une analyse des implications d'une didactique du plurilinguisme qui s'adapte à la diversité des contextes institutionnels et culturels et suggère des mesures (élaboration d'une politique linguistique d'établissement et construction curriculaire) pour la mise en œuvre concrète d'un enseignement plurilingue contextualisé et à géométrie variable exploitant la multiplicité des approches existantes.

Il testo enuncia le ragioni che giustificano l'insegnamento plurilingue nella scuola primaria, propone una analisi di ciò che implica una didattica del plurilinguismo che si adatti alla diversità dei contesti istituzionali e culturali e suggerisce alcune misure (elaborazione di una politica linguistica di istituto e costruzione curricolare) per una messa in opera di un insegnamento plurilingue contestualizzato e a geometria variabile che sfrutti la molteplicità degli approcci esistenti.

\section{INDEX}

Mots-clés : approches plurielles, construction curriculaire, plurilinguisme, politique linguistique d'établissement 


\section{AUTEUR}

\section{MARISA CAVALLI}

Enseignante chargée de recherche, Aire de la recherche éducative, Ex Institut Régional de Recherche Éducative pour le Val d'Aoste 